\title{
Somatosensory changes in Chinese patients after coronectomy vs. total extraction of mandibular third molar: a prospective study
}

\author{
Zi-Yu Yan ${ }^{1} \cdot$ Xiao-Yan Yan ${ }^{2} \cdot$ Chuan-Bin Guo ${ }^{1} \cdot$ Qiu-Fei Xie ${ }^{3} \cdot$ Guang-Ju Yang $^{3} \cdot$ Nian-Hui Cui ${ }^{4}$
}

Received: 20 September 2019 / Accepted: 25 November 2019 / Published online: 18 December 2019

(C) The Author(s) 2019

\begin{abstract}
Objectives This study aimed to quantitatively compare the somatosensory function changes of inferior alveolar nerve (IAN) after mandibular third molar extraction with a surgery protocol of coronectomy, as opposed to the conventional method.

Materials and methods Patients with a lower third molar directly contacting IAN were recruited and assigned either to a test group (coronectomy group) or a control group (conventional extraction). A standardized quantitative sensory testing (QST) battery was performed for four times: one week before surgery and the second, seventh, and 28th days after surgery. Z-scores and the loss/gain coding system were applied for each participant.

Results A total of 140 molars (test group: $n=91$, control group: $n=49$ ) were enrolled. The sensitivity of the mechanical detection threshold (MDT) and pressure pain threshold (PPT) significantly increased after surgery more than before surgery in both groups $(P \leq 0.001)$. After the surgery, the sensitivities of the cold detection threshold (CDT), cold pain threshold (CPT), and heat pain threshold (HPT) were significantly higher in the test group than in the control group $(P \leq 0.027)$. The risk of IANI was significantly larger $(P=0.041)$ in the test group than in the control group.

Conclusions QST was a sensitive way to detect somatosensory abnormalities even with no subjective complaint caused by surgery. Coronectomy had less influence on IAN function than conventional total extraction.

Clinical relevance The somatosensory function changes after mandibular third molar extraction were quantitatively studied, and coronectomy was proved a reliable alternation to reduce IAN injury rate.
\end{abstract}

Keywords Coronectomy $\cdot$ Inferior alveolar nerve injury $\cdot$ Quantitative sensory testing $\cdot$ Somatosensory function

Guang-Ju Yang

Nian-Hui Cui

drcuinianhui@163.com

1 Department of Oral and Maxillofacial Surgery, Peking University School and Hospital of Stomatology, 22 South Street Zhong Guan Cun, Haidian District, Beijing 100081, China

2 Department of Medical Statistics, Peking University Clinical Research Institute, Beijing, China

3 Department of Prosthodontics and Center for Oral Functional Diagnosis, Treatment and Research, Peking University School and Hospital of Stomatology, Beijing, China

4 Department of Oral and Maxillofacial Surgery Peking University School and Hospital of Stomatology \& National Clinical Research Center for Oral Diseases \& National Engineering Laboratory for Digital and Material Technology of Stomatology \& Beijing Key Laboratory of Digital Stomatology, Beijing 100081, China

$\begin{array}{ll}\text { Abbreviations } \\ \text { CBCT } & \text { cone-beam computed tomography } \\ \text { CDT } & \text { cold detection threshold } \\ \text { CNTs } & \text { clinical neurosensory tests } \\ \text { CPT } & \text { cold pain threshold } \\ \text { DFNS } & \text { the German Research Network on Neuropathic Pain } \\ \text { DMA } & \text { dynamic mechanical allodynia } \\ \text { HPT } & \text { heat pain threshold } \\ \text { IAN } & \text { inferior alveolar nerve } \\ \text { IANI } & \text { inferior alveolar nerve injury } \\ \text { MDT } & \text { mechanical detection threshold } \\ \text { MPS } & \text { mechanical pain sensitivity } \\ \text { MPT } & \text { mechanical pain threshold } \\ \text { NS } & \text { no significant difference } \\ \text { PHSs } & \text { paradoxical heat sensations } \\ \text { PPT } & \text { pressure pain threshold } \\ \text { QST } & \text { quantitative sensory testing } \\ \text { RCTs } & \text { randomized controlled trials } \\ \text { SD } & \text { standard deviation }\end{array}$


TSL thermal sensory limen

VAS visual analogue scale

VDT vibration detection threshold

WDT warm detection threshold

WUR wind-up ratio

\section{Introduction}

The prevalence of mandibular third molar impaction is increasing, and inferior alveolar nerve injury (IANI) after tooth extraction, one of the most serious complications [1], is attracting more attention. The incidence of IANI in normal cases, using conventional surgery, is reported to be $0.35 \%-8.1 \%$ for temporary complications and $0.0145 \%-3.6 \%$ for permanent ones [1-4]. In high-risk cases, the rate is up to $35.64 \%[1,5,6]$.

Coronectomy is an alternative to conventional extraction surgery; it removes the crown only and intentionally leaves the root to reduce the compression or direct contact injury to the inferior alveolar nerve (IAN), which is regarded as the origin of inferior alveolar nerve injury (IANI) [7-9]. This technique was first described in 1984 by Ecuyer and Debien [10] and suggested as a way to prevent IANI in 1989 [7]. In 2004, Pogrel detailed the method and presented basic rules [9]. Many studies claimed that coronectomy was significantly safer than conventional extraction. However, only two studies were randomized controlled trials (RCTs) $[11,12]$ with high-quality evidence $[13,14]$. Most of these studies used clinical neurosensory tests (CNTs), that is, light touch sensation or two-point discrimination, to recognize IANI $[2,8,11,12]$. The sensitivity and specificity of these methods proved unsatisfactory and did not permit standard comparison among studies [15].

In 2006, the German Research Network on Neuropathic Pain (DFNS) established a standardized quantitative sensory testing (QST) protocol for examination and data analysis, which systematically evaluates thermal and mechanical somatosensory functions [16-18]. Compared with CNTs, QST has better reproducibility and sensitivity in diagnosing and grading nerve injuries [15]. It has been widely applied in the orofacial region [19-23], and somatosensory sensitivity changes related to IANI can also be quantitatively measured.

The aim of this study was to use a standardized QST protocol to assess somatosensory function changes and recovery duration after mandibular third molar extraction by coronectomy or conventional surgery.

\section{Materials and methods}

This is a prospective study of postoperative IAN function and other complications from two surgical procedures, coronectomy and conventional tooth extraction. The biomedical ethics committee of Peking University Hospital of
Stomatology (PKUSSIRB-201736080) granted ethical approval, and the research approach was pre-registered in the China Clinical Trial Center (ChiCTR1800014862). The study design was non-randomized controlled trials with an openlabel and endpoint blinded. The hypothesis of this study was that conventional extraction surgery impairs IAN somatosensory function more than coronectomy does.

\section{Participants}

From 2018 to 2019, Chinese individuals with an impacted lower third molar to be extracted were recruited from the Department of Oral and Maxillofacial Surgery of Peking University School and Hospital of Stomatology, China. A flow diagram appears in Fig. 1. Inclusion criteria comprised healthy males and females ranging from 18 to 40 years old; at least one root of the lower third molar directly contacted the IAN, proven by preoperative cone-beam computed tomography (CBCT); and the patients' physical condition was tolerant of surgery. Exclusion criteria were the following: presence of preexisting neurosensory disorders affecting IAN function; local susceptible factors, such as caries or periodontitis of the third molar, cystic or neoplastic conditions around the third molar; general systemic disease contributing to infection (e.g., diabetes, immunodeficiency); or history of radiotherapy or chemotherapy.

All participants signed informed consent. Group selection was based on individual preference under the principle of ethics. Those preferring coronectomy were included in the test group, whereas those choosing conventional extraction were gathered in the control group.

\section{Experiment protocols}

1. CBCT examination was applied three times. Preoperatively, CBCT was used to determine the relationship between the lower third molar and the IAN and measure the size of perforation in the inferior alveolar canal (IAC). The other two CBCT scans were taken on the day after surgery and six months postoperatively.

2. QST was applied on the skin overlying the mental foramina of the affected side four times: one week before surgery and on the second, seventh, and 28th days after surgery [24, 25].

3. A visual analogue scale (VAS) of $10 \mathrm{~cm}$ was applied to record postoperative pain. Pain scores ranged from 0 (no pain) to 10 (most severe pain).

\section{Surgical technique}

All operations were performed under local anesthesia, by one experienced oral surgeon. During coronectomy, we applied a partial crown section (more than three quarters in depth) 1$2 \mathrm{~mm}$ below the cementoenamel junction and then separated them with an elevator [4]. The surface of the root was trimmed 
Fig. 1 Flow diagram

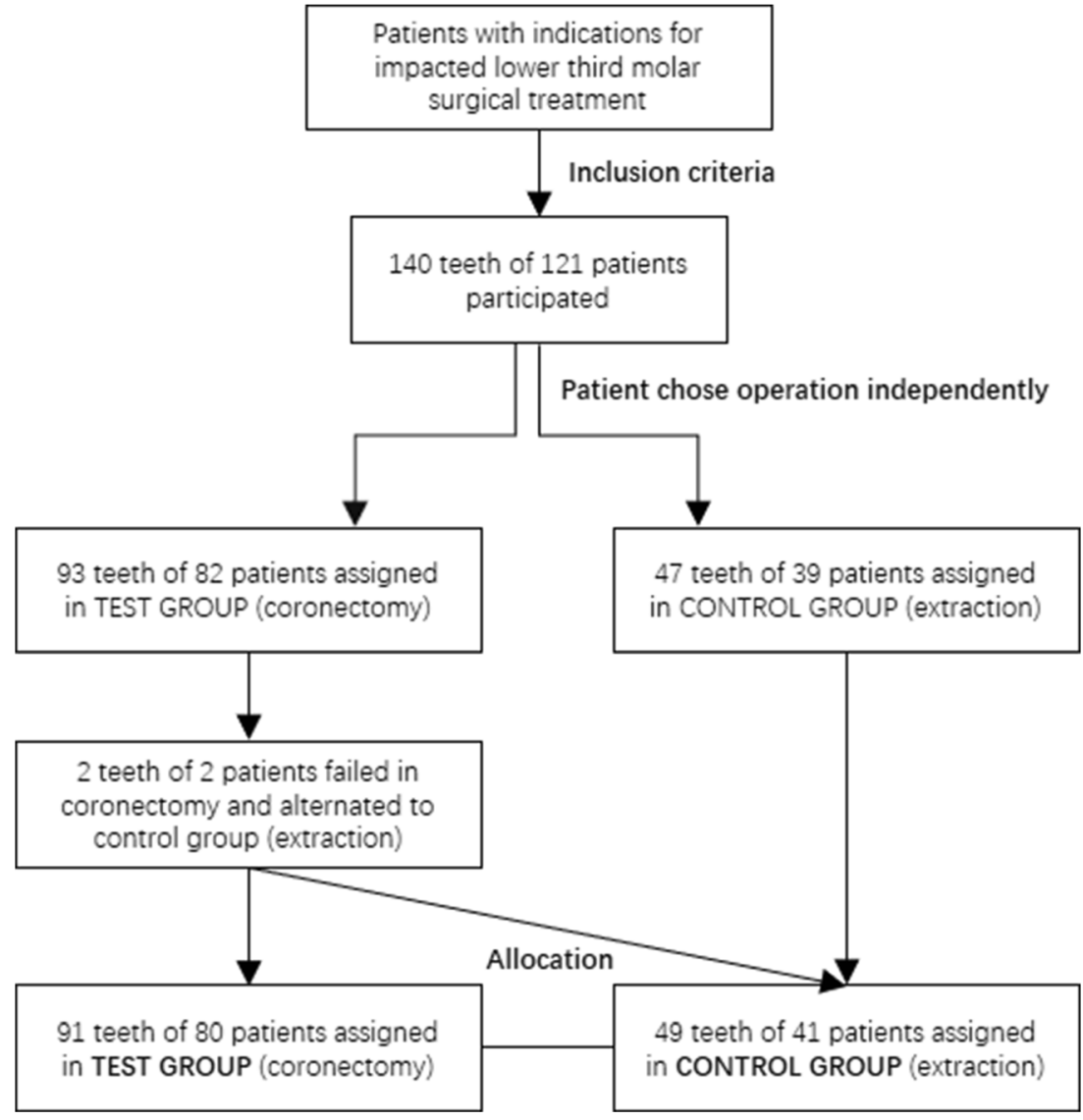

to at least $3 \mathrm{~mm}$ below the surrounding alveolar bone [9]. Unsuccessful coronectomy was defined as any evidence of root loosening $[9,12,26]$. After routine debridement and irrigation, the wound was primarily sutured. Conventional extraction was applied in the control group. Neither antibiotics nor analgesic was taken by either group.

\section{Quantitative sensory testing}

The standardized QST battery developed by DFNS [27-29] and modified for the trigeminal region [29-32] was used in this study. All QST measures were performed in a quiet room with approximate temperature between 21 and $23{ }^{\circ} \mathrm{C}$. The QST consisted of seven tests, measuring a total of 13 thermal and mechanical parameters. (A) Thermal testing comprised detection and pain thresholds for cold, warm, and hot stimuli (C- and A-delta fiber mediated): cold detection threshold (CDT); warm detection threshold (WDT); number of paradoxical heat sensations (PHSs) during the thermal sensory limen (TSL) procedure for alternating warm and cold stimuli; cold pain threshold (CPT); and heat pain threshold (HPT). (B) Mechanical detection threshold (MDT) was used as a test for A-beta fiber function, using von Frey filaments. (C) The mechanical pain threshold (MPT) was used as a test for A-delta fiber-mediated hyperor hypoalgesia to pinprick stimuli. (D) Stimulus-response functions were tested with mechanical pain sensitivity (MPS) for pinprick stimuli, and dynamic mechanical allodynia (DMA) assessed A-delta-mediated sensitivity to sharp stimuli (pinprick) and A-beta fiber-mediated pain sensitivity to stroking light touch (CW, cotton wisp; QT, cotton wool tip; BR, brush). (E) Wind-up ratio (WUR) compared the numerical ratings within three trains of a single pinprick stimulus (a) with a series (b) of 10 repetitive pinprick stimuli to calculate WUR as the ratio: b/a. (F) Vibration detection threshold (VDT) tested for A-beta fiber function, using a Rydel-Seiffer $64 \mathrm{~Hz}$ tuning fork. (G) Pressure pain threshold (PPT) was the only test for deep pain sensitivity, most probably mediated by muscle C- and A-delta fibers [16, 29, 31]. The investigator in this study was carefully instructed and trained under supervision, according to the latest guidelines [29, 32]. A standard set of instructions, lasting $1 \mathrm{~min}$, was read to the participants for each modality just before the beginning of each test; that is, there were one-minute intervals between tests $[16,29,33,34]$.

\section{Thermal thresholds and thermal sensory limen}

Thermal testing was performed using Medoc Pathway (Medoc Ltd., Israel) with an advanced thermal stimulator $(30 \mathrm{~mm} \times 30 \mathrm{~mm})[16,29,33,34]$. CDT, WDT, CPT, and HPT were measured in triplicate $[16,29,33,34]$. For the TSL, the temperature first went up, and the participants pressed a button when they perceived a change [16, 29, 33, 
34]. The number of PHSs during this procedure was recorded $[16,29,33,34]$. Baseline temperature was set at $32{ }^{\circ} \mathrm{C}$ for all thermal testing, ramped stimuli of $1{ }^{\circ} \mathrm{C} / \mathrm{s}$ was used, and cutoff temperatures were set at 0 and $50^{\circ} \mathrm{C}[16,29,33,34]$.

\section{Mechanical detection threshold}

The MDT was measured with a standard set of SemmesWeinstein monofilaments (Touch Test ${ }^{\mathrm{TM}}$ Sensory Evaluator, North Coast Medical, Inc., Morgan Hill, CA) with 20 diameters [29, 33-35]. Five repeated threshold measurements were made, each by applying a series of ascending and descending stimulus intensities; the final threshold was the geometric mean of the five series $[16,29,33,34]$.

\section{Mechanical pain threshold, mechanical pain sensitivity to pinprick stimuli, dynamic mechanical allodynia, and wind-up ratio for repetitive pinprick stimuli}

Weighted pinprick stimuli were delivered with seven custommade punctate mechanical stimulators with fixed stimulus intensities (flat contact area $0.2 \mathrm{~mm}$ in diameter) that exerted forces of $8-512 \mathrm{mN}$ to determine the MPT [16, 29, 33, 34]. The method of limits, which was used to determine the MDT, was also used to determine the MPT [16, 29, 33, 34]. MPS and DMA were evaluated using two sets of instruments in a stimulus-response assessment [16, 29, 36]. To determine MPS, seven weighted pinprick stimulators were used (as for MPT). Three tactile stimulators were used to determine DMA: a cotton wisp $(\sim 3 \mathrm{mN})$, a cotton wool tip (Q-tip, $\sim 100 \mathrm{mN}$ ) attached to a flexible handle, and a disposable toothbrush (Top Dent ${ }^{\circ}$, Meda AB, Solna, Sweden, $\sim 200-400 \mathrm{mN}$ ). A series of 10 measurements was made three times, each with 10 stimulators (seven pinpricks and three tactile stimulators) applied in a different order, as specified in the DFNS protocol [16,29, $33,34]$. For each of the resulting 30 stimuli, the participant chose a pain rating on a 0 to 100 scale with the endpoints 0 indicating "no pain" and 100 indicating "most intense pain imaginable."

To measure the WUR for repetitive pinprick stimuli, the perceived magnitude of a train of 10 pinprick stimuli repeated at $1 \mathrm{~Hz}$ was divided by that of a single pinprick stimulus with the same force $[16,29,33]$. The WUR test was repeated three times $[16,29,33,34]$.

\section{Vibration detection threshold}

The vibration detection threshold (VDT) was measured with a tuning fork (64 Hz, 8/8 scale) [16, 29, 36]. VDT was performed on bony prominences bilaterally for each participant: the zygomatic process, the lower edge of the mandible, and the ulnar styloid process. The participant indicated when the vibration could no longer be sensed on a nine-point $(0-8)$ scale $[16,29,33-35]$. The test was repeated three times.

\section{Pressure pain threshold}

The pressure pain threshold (PPT) was measured using a computerized pressure algometer (Medoc AlgoMed, Israel) [16, $29,33,34]$. PPT was measured on the painful site, the corresponding contralateral site, and the right thenar muscle of patients; and on the temporalis, masseter, and thenar muscles bilaterally of healthy participants, both with a constant application rate of $30 \mathrm{kPa} / \mathrm{s}[16,29,33,34]$. The test was repeated three times.

\section{Data analysis and statistics}

\section{Z-transformation of QST data}

For all 13 parameters, the recently published reference data for Chinese of both genders and three body regions (two facial sites and hands) were available as reference values for two age groups $[28,29]$.

Data of cold and heat pain thresholds and vibration detection thresholds were normally distributed. Other parameters were log-transformed before analysis [27-29]. Each variable of individual QST data was $Z$-transformed based on reference data: $Z=\left(\mathrm{X}_{\text {single patient }}-\mathrm{Mean}_{\text {reference }}\right) / \mathrm{SD}_{\text {reference }}[16,27$, 29]. The age group, gender, and site-stratified data were the sign of the resulting $Z$-score and adjusted in such a way that those $>0$ indicated a gain of function when the participant was more sensitive to the stimuli than were controls (hyperesthesia, hyperalgesia, and allodynia). $Z$-scores $<0$ indicated a loss of function, referring to a lower sensitivity (hypoesthesia and hypoalgesia) $[23,29,36]$. $Z$-scores $>1.96$ and $<-1.96$ indicated values outside of the $95 \% \mathrm{CI}$ of the reference group data. Such values were considered abnormalities $[16,29,36]$.

\section{Interpretation of sensory loss and gain}

The loss/gain coding system was applied [23, 29, 36]. The loss/gain score combines a score of somatosensory loss of function (L0, L1, L2, or L3) with a score of somatosensory gain of function (G0, G1, G2, or G3) $[23,29,36]$. The number after the letter $\mathrm{L}$ or $\mathrm{G}$ indicates whether the abnormality is related to the thermal modalities alone (1), the mechanical modalities alone (2), or mixed (3) thermal and mechanical. If measures of thermal and/or mechanical detection (CDT, WDT, TSL, MDT, or VDT) were abnormal on the affected side in comparison with the reference data (absolute abnormality), or if abnormally large preoperative and postoperative differences were detected (relative abnormality), they were recorded as one of the following: L1, isolated loss of small fiber function (if abnormal thermal detection thresholds [CDT, 
TSL, or WDT] alone); L2, isolated loss of large fiber function (if abnormal mechanical detection thresholds [MDT or VDT] alone); or L3, mixed loss of function (if loss of both small and large fiber function) $[23,29,36]$. Likewise, for somatosensory gain, thermal hyperalgesia (G1) was recorded if gain of function in cold or heat pain thresholds (CPT or HPT) were found (absolute or relative abnormality). Mechanical hyperalgesia (G2) was recorded if gain of function (absolute or relative abnormality) was detected for MPT, MPS, or PPT, or if the DMA score exceeded zero. Mixed gain (G3) was recorded in individuals with gain of both thermal and mechanical somatosensory function. L0 was scored if no loss of somatosensory function was presented, and G0 if no gain of somatosensory function was detected.

\section{Statistical analysis}

All statistical analyses were performed using SPSS 21.0 software. Age and size of perforation in IAC differences between groups were compared using an unpaired $t$ test. Operation time and postoperative pain differences between groups were analyzed using a Mann-Whitney $U$ test. The distribution of gender and frequencies of loss and gain function according to the loss/gain coding system differences between groups were evaluated with Chi-square and Fisher's exact tests.

The effects of four primary factors (gender, group, what time, and size of perforation in the IAC) on QST results were calculated using a four-way repeated-measure ANOVA [16, 28, 29]. If significant influence were found between size of perforation in the IAC and QST results, a linear regression model was applied to estimate further. The effect of other secondary factors (postoperative pain, infection rate) on QST values were analyzed with multiple factors ANOVA analysis. Multiple comparisons were calculated using a Bonferroni post hoc test. In addition, for each surgical method, the variation of QST data and pain scores were analyzed with the Friedman test. Statistically significant difference was estimated with a value of $P<0.05$.

\section{Results}

A total of 140 impacted lower third molars of 121 patients were assigned to two groups. A total of 91 teeth were allocated the test group, and the other 49 teeth were included in the control group (Table 1). No significant age or gender differences were detected in the two groups.

\section{Clinical assessments}

The size of perforation in the inferior alveolar canal (IAC) in the test group $(5.87 \pm 2.82 \mathrm{~mm}$, mean $\pm \mathrm{SD})$, which was measured on CBCT, was higher than that of the control group $(4.86 \pm 2.63 \mathrm{~mm})(P=0.041)$.

Length of operation for coronectomy $(13.89 \pm 5.17 \mathrm{~min})$ was significantly greater than for traditional extraction $(12.65 \pm 4.68 \mathrm{~min})(P=0.015)$.

Two patients $(2.20 \%)$ in the test group complained of lower-lip numbness; only one patient in the control group complained of tongue numbness $(2.04 \%)$ (control vs. test group, $P=0.951)$.

There was no significant difference in postoperative pain rating or infection rate between the two groups $(P \geq 0.196)$. No suspicious pulpitis or root-related infection was found. The occurring of infection or dry socket, following treatment and recovery, was basically similar in two groups. No significant difference between infection and QST parameters changes was found because the sample of infection was too small.

\section{QST results}

No PHS or DMA was found in this study. The effect of factors (gender, group, test time, and size of perforation in the IAC) on QST results was assessed by four-way repeated-measure ANOVA (Table 2).

Significant gender differences were detected for most QST parameters except for WUR, with females more sensitive than males ( $P \leq 0.046$, Table 2), with an exception for VDT (males were more sensitive than females, $P=0.003$ ).

There were no group differences in any QST parameters before surgery $(P \geq 0.128)$. Postoperatively, however, in thermal parameters for the test group, CDT $\left(-0.48 \pm 0.20^{\circ} \mathrm{C}\right)$, CPT $\left(25.26 \pm 6.64{ }^{\circ} \mathrm{C}\right)$, and HPT $\left(37.93 \pm 3.39^{\circ} \mathrm{C}\right)$ were significantly more sensitive than the CDT $\left(-0.53 \pm 0.30^{\circ} \mathrm{C}, P=\right.$ $0.005)$, CPT (23.25 $\left.\pm 7.48^{\circ} \mathrm{C}, P=0.027\right)$, and HPT (38.52 \pm $3.30{ }^{\circ} \mathrm{C}, P=0.006$ ) in the control group. Furthermore, the mechanical parameter, PPT $(104.44 \pm 44.81 \mathrm{kPa}, P=0.004)$, in the test group was significantly more sensitive (lower threshold) than that $(112.92 \pm 39.72 \mathrm{kPa})$ in the control group.

There were significant time effects on the MDT and PPT $(P \leq 0.001)$ (Table 2$)$. The MDT before surgery $(0.14 \pm$ $0.09 \mathrm{mN}$ ) was higher (less sensitive) than that of seven $(0.12 \pm 0.08 \mathrm{mN})$ or 28 days $(0.11 \pm 0.07 \mathrm{mN})$ after surgery. The PPT one day $(98.16 \pm 46.96 \mathrm{kPa})$ after surgery was lower (more sensitive) than that preoperatively $(114 \pm 34.15 \mathrm{kPa})$ or seven $(112.22 \pm 40.94 \mathrm{kPa})$ or 28 days $(111.71 \pm 40.44 \mathrm{kPa})$ after surgery.

The effects of time on each QST parameter were also assessed in each group separately (Fig. 2). Significant time effects were detected for mechanical parameters (MDT, PPT) in both groups. The MDT seven days after surgery was lower (more sensitive) than that preoperatively or one day after surgery $(P \leq 0.016)$. The PPT one day after surgery was lower (more sensitive) than that of seven days after surgery 
Table 1 Patient characteristics and postoperative assessments

\begin{tabular}{|c|c|c|c|c|c|c|c|c|c|c|c|c|c|c|c|c|}
\hline & \multicolumn{2}{|c|}{ Sample size } & \multicolumn{2}{|c|}{$\begin{array}{l}\text { Gender (teeth } \\
\text { numb.) }\end{array}$} & \multicolumn{2}{|c|}{ Age (year) } & \multicolumn{2}{|c|}{$\begin{array}{l}\text { Perforation in } \\
\text { IAC }^{*}(\mathrm{mn})\end{array}$} & \multicolumn{2}{|c|}{$\begin{array}{l}\text { Operation time* } \\
\text { (min) }\end{array}$} & \multicolumn{2}{|c|}{ Pain (VAS) } & \multicolumn{2}{|c|}{ Infection rate } & \multicolumn{2}{|c|}{ Paresthesia } \\
\hline & Patient & Teeth & Male & Female & Mean & $\mathrm{SD}$ & Mean & $\mathrm{SD}$ & Mean & $\mathrm{SD}$ & Mean & $\mathrm{SD}$ & Number & Rate $(\%)$ & Number & Rate (\%) \\
\hline Test & 80 & 91 & 34 & 57 & 27.20 & 4.31 & 5.87 & 2.82 & 13.89 & 5.17 & 1.99 & 1.38 & $10 / 91$ & 10.99 & $2 / 91$ & 2.20 \\
\hline Control & 41 & 49 & 15 & 34 & 28.04 & 4.26 & 4.86 & 2.63 & 12.64 & 4.68 & 1.84 & 1.86 & $5 / 49$ & 10.20 & $1 / 49$ & 2.04 \\
\hline Total & 121 & 140 & 49 & 91 & 27.49 & 4.30 & 5.52 & 2.79 & 13.46 & 5.02 & 1.94 & 1.56 & $15 / 140$ & 10.71 & $2 / 140$ & 2.14 \\
\hline
\end{tabular}

Assessments that had significant difference in two groups were marked by *

SD, standard deviation; VAS, visual analogue scale; pain, postoperative pain, which was calculated as the average VAS score of postoperative 1st day, 3rd day, 5th day, and 7th day

$(P \leq 0.038)$. Significant time effects were detected for thermal parameters (CDT, HPT) only in the test group.

\section{Abnormalities based on Z-scores}

The abnormality included a relative $Z$-score (compared with data before surgery) and an absolute $Z$-score (compared with established age and gender-stratified data) for each QST parameter (Fig. 3). There was no significant group difference for abnormal frequencies between the two groups (rate for control group $\leq 22.14 \%$, rate for test group $\leq 37.14 \%, P \geq 0.17$ ), but mechanical parameters changed obviously. The abnormal frequency for MDT (preoperatively: 24.43\%) almost tripled one day after surgery $(59.29 \%)$ and then decreased apparently seven days after surgery (7.14\%), and the abnormal frequency for PPT varied in a similar way, with the result (4.29\%) greatly increasing one day after surgery $(22.14 \%)$ and then decreasing sharply seven days after surgery (5.71\%). Finally, 28 days after surgery, the abnormal frequency for PPT reverted to preoperative level $(5.71 \%)$ but the level for MDT did not completely recover (test $7.14 \%$ ). However, thermal parameters just showed a little variation during those periods.

Regarding the loss/gain coding system, all data was calculated without group separation (Table 3). Compared with other testing points $(62.20 \%$ preoperatively, $68.75 \%$ seven days postoperatively $72.26 \%, 28$ days postoperatively), the absence of somatosensory abnormalities (L0G0) significantly decreased one day after surgery $(34.88 \%, P<0.001)$. Change of mechanical sensory data was greater than thermal sensory data change. The cumulative proportion of those manifesting mechanical somatosensory loss (L2G0, L2G1, L2G2, L2G3) was significantly higher one day after surgery

Table 2 Four-way repeated-measure ANOVA analysis

\begin{tabular}{llllllllllllll}
\hline & CDT & WDT & TSL & PHS & CPT & HPT & MDT & MPT & MPS & DMA & WUR VDT & PPT \\
\hline ANOVA factors & & & & & & & & & & & & \\
1. Gender & $<0.001$ & $<0.001$ & $<0.001$ & NO & $<0.05$ & $<0.001$ & $<0.001$ & $<0.001$ & $<0.001$ & NO & NS & $<0.01$ & $<0.001$ \\
2 Group & $<0.01$ & NS & NS & NO & $<0.05$ & $<0.01$ & NS & NS & NS & NO & NS & NS & $<0.01$ \\
3. Time & NS & NS & NS & NO & NS & NS & $<0.001$ & NS & NS & NO & NS & NS & $<0.001$ \\
4. Size of perforation in IAC & NS & NS & NS & NO & NS & NS & NS & $<0.05$ & $<0.001$ & NO & NS & $<0.001$ & NS \\
$1 \times 2$ & $<0.05$ & NS & NS & NO & NS & $<0.01$ & $<0.05$ & NS & NS & NO & NS & NS & NS \\
$1 \times 3$ & NS & NS & NS & NO & NS & NS & NS & NS & NS & NO & NS & NS & NS \\
$2 \times 3$ & NS & NS & NS & NO & NS & NS & NS & NS & NS & NO & NS & NS & NS \\
$1 \times 2 \times 3$ & NS & NS & NS & NO & NS & NS & NS & NS & NS & NO & NS & NS & NS \\
Effective size of & & & & & & & & & & & & \\
Gender difference & 0.084 & 0.034 & 0.039 & NO & 0.008 & 0.041 & 0.398 & 0.035 & 0.038 & NO & 0.003 & 0.016 & 0.123 \\
Coronectomy vs. extraction & 0.015 & 0.005 & 0.002 & NO & 0.009 & 0.015 & 0.002 & 0.003 & 0.000 & NO & 0.001 & 0.001 & 0.017 \\
Testing point of QST & 0.006 & 0.004 & 0.007 & NO & 0.001 & 0.007 & 0.032 & 0.001 & 0.020 & NO & 0.009 & 0.005 & 0.049 \\
Risk of IANI & 0.002 & 0.007 & 0.001 & NO & 0.000 & 0.000 & 0.005 & 0.007 & 0.011 & NO & 0.006 & 0.031 & 0.002 \\
\hline
\end{tabular}

Four-way repeated-measure ANOVA and effect size of gender, group, time, and size of perforation in inferior alveolar canal (IAC) on quantitative sensory testing (QST) parameters in 140 participants. $P$ values were filled in blanks. "NS" means "no significant difference", which means $P>0.05$

NO, no occurrence; CDT, cold detection threshold; WDT, warmth detection threshold; TSL, thermal sensory limen; PHS, paradoxical heat sensation; CPT, cold pain threshold; HPT, heat pain threshold; MDT, mechanical detection threshold; MPT, mechanical pain threshold; MPS, mechanical pain sensitivity; DMA, dynamic mechanical allodynia; WUR, wind-up ratio; VDT, vibration detection threshold; PPT, pressure pain threshold 


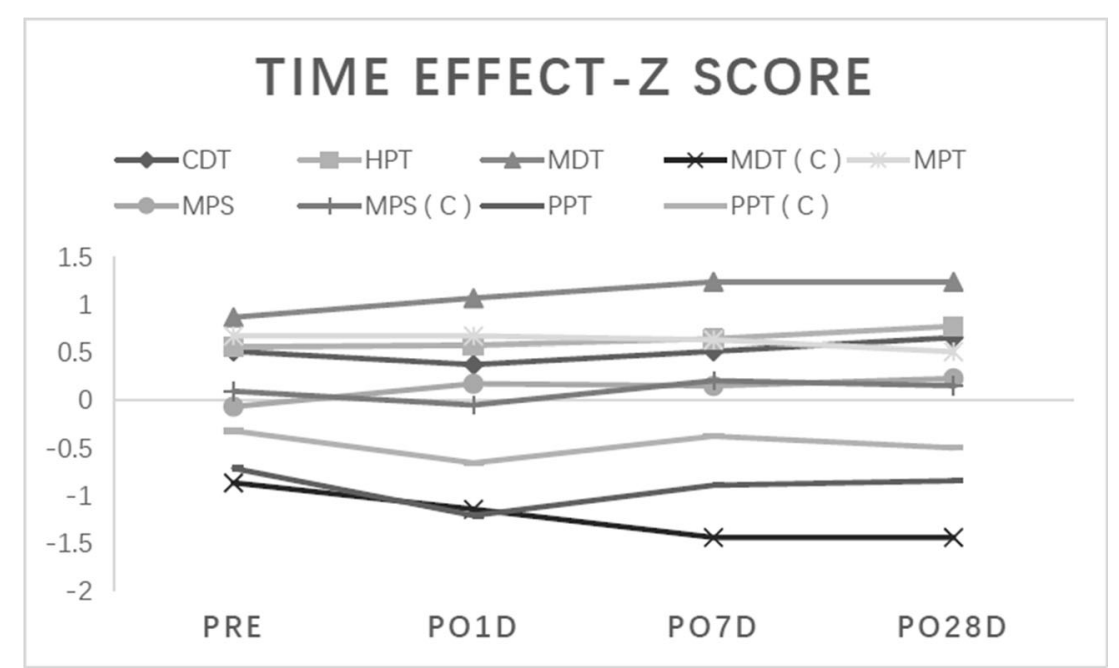

Fig. 2 Changing trend of quantitative sensory testing (QST) parameters which had significant time effects were showed. The $Z$-transformed values in the two groups were analyzed. "(C)" meant "control group". Without that sign meant the parameter was in test group. The vertical axis was the relative $Z$-transformed value. The value between -1.96 and 1.96

(42.64\%) than that of seven days $(16.41 \%)$ and 28 days $(14.60 \%)(P<0.001)$. Similarly, the cumulative proportion of those manifesting mechanical somatosensory gain (L0G2, L1G2, L2G2, L3G2) was significantly higher one day after surgery $(17.83 \%)$ than it was preoperatively $(0.00 \%)$, seven days $(1.56 \%)$, or 28 days $(2.92 \%)(P<0.001)$ after surgery (Table 3, light-gray shading). However, thermal somatosensory data exhibited little variation; no significant differences were found in the cumulative proportion of those exhibiting thermal somatosensory loss (L1G0, L1G1, L1G2, L1G3) or gain (L0G1, L1G1, L2G1, L3G1) among different testing time points $(P>0.05)$.

\section{Discussion}

This prospective study systematically compared the somatosensory profiles of two groups of patients with an impacted lower third molar extracted by one of two surgical methods, by using a comprehensive QST protocol for the first time [13, 14]. According to the previous studies, the Ethics Committee suggested that random grouping was not suitable for this study. In addition, participants with higher risk of IANI were more willing to join coronectomy group. It could account for the unbalanced sample size between two groups and the result that the size of perforation in IAC in the test group was significant higher than that of the control group $(P=0.041)$. However, this made it more conductive to demonstrate the superiority of coronectomy. The main finding of this study was that QST was a sensitive way to detect somatosensory abnormalities even with no subjective complaint caused by surgery. Coronectomy, which had less influence on the IAN meant that it was inside the reference 95\% confidence interval. "Pre" meant "Pre-operative", "Po" meant "Postoperative", "D" meant "Day". CDT, cold detection threshold; HPT, heat pain threshold; MDT, mechanical detection threshold; MPT, mechanical pain threshold; MPS, mechanical pain sensitivity; PPT, pressure pain threshold

somatosensory function in situations when the third molar contacted the IAN on CBCT, was a good alternative to conventional total extraction.

\section{QST is a sensitive way to detect somatosensory abnormalities}

The advantage of QST protocol was that it could be applied to identify many kinds of somatosensory abnormalities and help elucidate various mechanisms of nerve injury [16]. This protocol had already been widely used in the oral facial region [19-23]. In this study, most participants did not complain of subjective paresthesia of the lower lip, but significant variations were detected on QST values; for example, according to the loss/gain coding system, the absence of somatosensory abnormalities (L0G0) decreased significantly after surgery $(P<0.001)$, which was consistent with previous studies of implant surgery and third molar extraction [21, 22, 24, 25]. In addition, patients underwent minor orofacial surgery with no subjective somatosensory changes, which could present both central and peripheral sensitizations [25] identified by a similar QST protocol.

In this study, the sensitivity of mechanical parameters exhibited a significant increase after surgery (MDT and PPT, $P \leq 0.001)$, whereas the sensitivity of thermal parameters showed little variation $(P>0.05)$. The same results were also determined in the loss/gain coding system, in which only the frequency of mechanical somatosensory abnormalities varied significantly $(P<0.001)$. A previous study reported that mechanical tests yielded more abnormalities than thermal tests in patients with sensory abnormalities [36]. Hyperalgesia to pressure stimulus, evaluated by PPT, was probably caused 


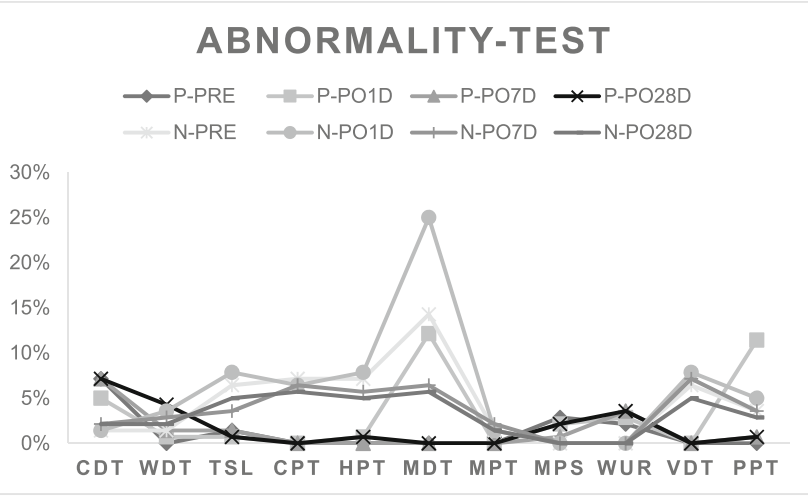

ABNORMALITY-CONTROL

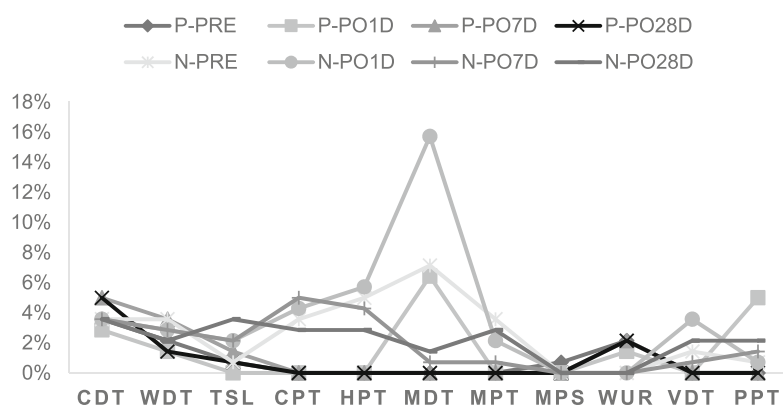

Fig. 3 Abnormality percentage in two groups. The frequency of patients presenting with $Z$-score values outside the reference $95 \%$ confidence interval was showed $(-1.96<Z$ score $<1.96)$. "P" meant "positive abnormality" $(1.96<Z$ score), "N" meant "negative abnormality" $(Z$ score $<-1.96)$. "D" meant "day". Regardless of a relative $Z$-score abnormality (compared with data before surgery) or an absolute $Z$-score abnormality (compared with established age and gender-stratified data), each QST parameter would be determined as abnormality as long as either of them was met. The vertical axis was the percentage of abnormality in each group. CDT, cold detection threshold; WDT, warmth detection threshold; TSL, thermal sensory limen; CPT, cold pain threshold; HPT, heat pain threshold; MDT, mechanical detection threshold; MPT, mechanical pain threshold; MPS, mechanical pain sensitivity; WUR, wind-up ratio; VDT, vibration detection threshold; PPT, pressure pain threshold

by postoperative inflammation and swelling of surrounding soft tissue and nerve fibers, which aggravated to maximum within $24 \mathrm{~h}$ and exactly met the lowest threshold (most sensitive) of PPT one day after surgery in both groups $[19,37]$. The sensitivity of MDT $(P=0.001)$ was significantly higher (lower threshold) after surgery than at the preoperative baseline. This was contrary to many studies, which reported that the sensitivity of MDT would decrease after surgery compared with before surgery [20,21, 23, 36, 38]. Controversial results were found in QST tests after surgery [23]. Huang found in an animal operative injury model that somatosensory changes varied in different situations [39]; a light compression on the right C7 dorsal root of Sprague-Dawley rats will lead to hyperalgesia, whereas a heavier force may induce hypoalgesia. That means as the nerve damage increases, the neurological function manifests a sensitivity increase at first and then a sensitivity decrease if the injury continues increasing. In this study, the increased sensitivity of MDT may be an indicator of limited deficits in the IAN sensory system.

Decreased sensitivity of thermal parameters was reported in many previous studies [21, 40-42]. The CDT and WDT are innervated by C-fibers [27], which are sensitive to nerve injury. In Kim HK's study, CDT and WDT showed a mild to high positive correlation with subjective symptoms, and WDT was a key indicator of permanent subjective paresthesia [40]. Once injured, WDT would exhibit an increased threshold (decreased sensitivity), and its recovery speed would be the slowest among all QST parameters [40, 43]. Almost no participants complained of subjective paresthesia, and the recovery speed of thermal somatosensory abnormalities was faster than mechanical ones. It was reasonable to assume that the damage to the IAN was limited, not sufficient to induce subjective symptoms and significant abnormality evaluated by the WDT. In addition, surgical procedures may lead to homogeneous nerve fiber damage [44]. As we discussed earlier, if surgery damaged the nerve function seriously, the sensitivity of the MDT would decrease, which meant that the increased sensitivity of MDT after surgery also confirmed that the influence on the IAN was not enough to decrease the sensitivity of the MDT. The greater variation of the MDT before and after surgery, compared with thermal parameters, indicated that it was more sensitive to surgical injury than thermal thresholds were, as well as a more sensitive indicator of recovery [20, 45].

Coronectomy is a better choice than conventional extraction when the root is close to the nerve.

IANI often resulted from direct compression, such as pressure from the tooth's root and surgical instruments [8]. The nerve injury rate would increase $20 \%$ if exposure of the IAN was larger than $3 \mathrm{~mm}$ [46], which meant the size of perforation in the IAC could indicate the risk of IANI. In this study, the size of perforation in the IAC was significantly larger $(P=$ $0.041)$ in the test group $(5.87 \pm 2.82 \mathrm{~mm})$ than in the control group $(4.86 \pm 2.63 \mathrm{~mm})$, but no significant difference $(P>0.05)$ in abnormal frequencies of QST values was found between the two groups after surgery. Thus, coronectomy had less influence on IAN function than conventional extraction when tooth roots contacted the IAN directly. This was consistent with previous studies. Long H's systematic review indicated that the risk of incurring IANI during conventional extraction would be nearly 10 times higher than during coronectomy [13], and the safety of coronectomy had also been reported in many qualitative studies [1, 12, 47].

Different injury led to different manifestation [39]; this study provided more detail about the difference. CDT, HPT only exhibited the time effect in the test group $(P \leq 0.036)$ and, after surgery, CDT, CPT, and HPT in the coronectomy group were significantly more sensitive than those in the extraction group $(P \leq 0.027)$. Thermal parameters seemed to be more 
Table 3 Results of loss/gain coding system

\begin{tabular}{|c|c|c|c|c|c|c|c|c|c|c|c|}
\hline \multirow[t]{2}{*}{ Loss } & & \multicolumn{10}{|l|}{ Gain } \\
\hline & & G0 & (No) & G1 & (thermal) & $\mathrm{G} 2$ & (mechanical) & G3 & (both) & & All \\
\hline \multicolumn{12}{|c|}{ Pre-operation } \\
\hline L0 & (No) & 79 & $62.20 \%$ & 0 & $0.00 \%$ & 0 & $0.00 \%$ & 0 & $0.00 \%$ & 79 & $62.20 \%$ \\
\hline L1 & (thermal) & 11 & $8.66 \%$ & 0 & $0.00 \%$ & 0 & $0.00 \%$ & 0 & $0.00 \%$ & 11 & $8.66 \%$ \\
\hline L2 & (mechanical) & 35 & $27.56 \%$ & 0 & $0.00 \%$ & 0 & $0.00 \%$ & 0 & $0.00 \%$ & 35 & $27.56 \%$ \\
\hline L3 & (both) & 2 & $1.57 \%$ & 0 & $0.00 \%$ & 0 & $0.00 \%$ & 0 & $0.00 \%$ & 2 & $1.57 \%$ \\
\hline \multicolumn{2}{|c|}{ All } & 127 & $100.00 \%$ & 0 & $0.00 \%$ & 0 & $0.00 \%$ & 0 & $0.00 \%$ & 127 & $100.00 \%$ \\
\hline \multicolumn{12}{|c|}{ Postoperative 1st day } \\
\hline L0 & (No) & 45 & $34.88 \%$ & 1 & $0.78 \%$ & 11 & $8.53 \%$ & 1 & $0.78 \%$ & 58 & $44.96 \%$ \\
\hline L1 & (thermal) & 11 & $8.53 \%$ & 0 & $0.00 \%$ & 2 & $1.55 \%$ & 0 & $0.00 \%$ & 13 & $10.08 \%$ \\
\hline L2 & (mechanical) & 45 & $34.88 \%$ & 0 & $0.00 \%$ & 10 & $7.75 \%$ & 0 & $0.00 \%$ & 55 & $42.64 \%$ \\
\hline L3 & (both) & 3 & $2.33 \%$ & 0 & $0.00 \%$ & 0 & $0.00 \%$ & 0 & $0.00 \%$ & 3 & $2.33 \%$ \\
\hline \multicolumn{2}{|c|}{ All } & 104 & $80.62 \%$ & 1 & $0.78 \%$ & 23 & $17.83 \%$ & 1 & $0.78 \%$ & 129 & $100.00 \%$ \\
\hline \multicolumn{12}{|c|}{ Postoperative 7 th day } \\
\hline L0 & (No) & 88 & $68.75 \%$ & 0 & $0.00 \%$ & 2 & $1.56 \%$ & 0 & $0.00 \%$ & 90 & $70.31 \%$ \\
\hline L1 & (thermal) & 14 & $10.94 \%$ & 0 & $0.00 \%$ & 0 & $0.00 \%$ & 0 & $0.00 \%$ & 14 & $10.94 \%$ \\
\hline L2 & (mechanical) & 21 & $16.41 \%$ & 0 & $0.00 \%$ & 0 & $0.00 \%$ & 0 & $0.00 \%$ & 21 & $16.41 \%$ \\
\hline \multirow[t]{2}{*}{ L3 } & (both) & 3 & $2.34 \%$ & 0 & $0.00 \%$ & 0 & $0.00 \%$ & 0 & $0.00 \%$ & 3 & $2.34 \%$ \\
\hline & All & 126 & $98.44 \%$ & 0 & $0.00 \%$ & 2 & $1.56 \%$ & 0 & $0.00 \%$ & 128 & $100.00 \%$ \\
\hline \multicolumn{12}{|c|}{ Postoperative 28th day } \\
\hline L0 & (No) & 99 & $72.26 \%$ & 1 & $0.73 \%$ & 4 & $2.92 \%$ & 0 & $0.00 \%$ & 104 & $75.91 \%$ \\
\hline L1 & (thermal) & 11 & $8.03 \%$ & 0 & $0.00 \%$ & 0 & $0.00 \%$ & 0 & $0.00 \%$ & 11 & $8.03 \%$ \\
\hline L2 & (mechanical) & 20 & $14.60 \%$ & 0 & $0.00 \%$ & 0 & $0.00 \%$ & 0 & $0.00 \%$ & 20 & $14.60 \%$ \\
\hline L3 & (both) & 2 & $1.46 \%$ & 0 & $0.00 \%$ & 0 & $0.00 \%$ & 0 & $0.00 \%$ & 2 & $1.46 \%$ \\
\hline \multicolumn{2}{|c|}{ All } & 132 & $96.35 \%$ & 1 & $0.73 \%$ & 4 & $2.92 \%$ & 0 & $0.00 \%$ & 137 & $100.00 \%$ \\
\hline
\end{tabular}

This table exhibited LG rates of 140 participants on 4 different time points. The number of samples and the percentage of it were filled in order. L0, no loss of detection; L1, only thermal loss; L2, only mechanical loss; L3, mixed loss of detection; G0, no gain (= no hyperalgesia); G1, with only thermal hyperalgesia; G2, with only mechanical hyperalgesia; G3 with both thermal and mechanical hyperalgesia. The light-gray shading area emphasized cumulative proportion changes of the absence of somatosensory abnormalities (L0G0), mechanical somatosensory loss (L2G0, L2G1, L2G2, L2G3) and mechanical somatosensory gain (L0G2, L1G2, L2G2, L3G2)

effective in reflecting postoperative IAN function in coronectomy. Retained dental pulp was probably an important factor contributing to this phenomenon. It was reported that dental pulp would retain vitality after coronectomy [48, 49]. Neurogenic inflammation after pulpectomy might lead to higher sensitivity to thermal stimulus [50]. The hypoalgesia to thermal stimuli, HPT and CPT, caused by surgery may recover slower than the MDT [40]. The current study obtained a contrary result regarding different thermal parameters, indicating that the variation of thermal parameters might be motivated by pulpectomy, leading to peripheral sensitization of cold fibers (CPT) and heat hyperalgesia [42, 51].

There was no difference between the two groups in the incidence of postoperative complications (pain, dry sockets, and infection) $(P \geq 0.196)$, which was consistent with previous studies $[13,26]$. Dry socket is microscopically characterized as osteomyelitis and macroscopically has many similarities to infection [52]. Dry socket and infection may impair IAN function similarly through inflammation, so we counted the two complications together during analysis. There was no significant correlation between infection and QST results. The operation time for coronectomy $(13.89 \pm 5.17 \mathrm{~min})$ was about 1 min longer than conventional extraction $(12.65 \pm 4.68 \mathrm{~min})$ and was within acceptable time; it could hardly lead to significant nerve damage.

The failure rate of coronectomy was $2.1 \%$ (2/94), which was lower than previous reports, $2.3 \%-38 \%$ [12, 26, 49]. Among the infected cases, all participants recovered through conservative treatment, which indicated that the retained fragment of roots were not involved and did not require further operation [26]. Unfortunately, the follow-up time in our study was too short to propose a conclusion on root prognosis. The migration of retained root rates is reported to be from $14 \%$ to $81 \%[26,53,54]$. We just presented a case with a follow-up time of more than six months to draw a general impression of later prognosis that is safe and good. Subsequent results will be presented in our further report.

\section{Conclusion}

This prospective study on postoperative IAN function demonstrated that coronectomy had less influence on IAN function and was a good alternative to conventional total extraction when the third molar contacted the IAN directly. QST was a 
sensitive way to detect somatosensory abnormalities even with no subjective complaint caused by surgery. Mechanical parameters, especially MDT, were a sensitive indicator of both injury and recovery. Thermal parameters, CDT, CPT, and HPT, were probably more suitable to reflect IAN condition after coronectomy.

Acknowledgment We appreciated the patience and cooperation of all the participants involved in this study. The authors gratefully acknowledge Prof. En-Bo Wang, Prof. Yu Liu, Prof. Na Ge, Prof. Xiang-Liang Xu, Prof. Deng-Hui Duan, Dr. Jing Wang, and other staff from the Department of Oral and Maxillofacial Surgery, Peking University School and Hospital of Stomatology, for their efforts on participant recruitment.

Funding This research did not receive any specific grant from funding agencies in the public, commercial, or not-for-profit sectors.

\section{Compliance with ethical standards}

Conflict of interest $\mathrm{Zi}-\mathrm{Yu}$ Yan declares that he has no conflict of interest. Xiao-Yan Yan declares that she has no conflict of interest. Chuan-Bin Guo declares that he has no conflict of interest. Qiu-Fei Xie declares that she has no conflict of interest. Guang-Ju Yang declares that he has no conflict of interest. Nian-Hui Cui declares that he has no conflict of interest.

Ethical approval All procedures performed in studies involving human participants were in accordance with the ethical standards of the institutional and/or national research committee and with the 1964 Helsinki declaration and its later amendments or comparable ethical standards.

Informed consent Informed consent was obtained from all individual participants included in the study.

Open Access This article is licensed under a Creative Commons Attribution 4.0 International License, which permits use, sharing, adaptation, distribution and reproduction in any medium or format, as long as you give appropriate credit to the original author(s) and the source, provide a link to the Creative Commons licence, and indicate if changes were made. The images or other third party material in this article are included in the article's Creative Commons licence, unless indicated otherwise in a credit line to the material. If material is not included in the article's Creative Commons licence and your intended use is not permitted by statutory regulation or exceeds the permitted use, you will need to obtain permission directly from the copyright holder. To view a copy of this licence, visit http://creativecommons.org/licenses/by/4.0/.

\section{References}

1. Moreno-Vicente J, Schiavone-Mussano R, Clemente-Salas E, Marí-Roig A, Jané-Salas E, López-López J (2015) Coronectomy versus surgical removal of the lower third molars with a high risk of injury to the inferior alveolar nerve. A bibliographical review. Med Oral Patol Oral Cir Bucal 20(4):e508-e517

2. Robinson PP, Loescher AR, Yates JM, Smith KG (2004) Current management of damage to the inferior alveolar and lingual nerves as a result of removal of third molars. Br J Oral Maxillofac Surg 42(4):285-292. https://doi.org/10.1016/j.bjoms.2004.02.024
3. Cheung LK, Leung YY, Chow LK, Wong MC, Chan EK, Fok YH (2010) Incidence of neurosensory deficits and recovery after lower third molar surgery: a prospective clinical study of 4338 cases. Int $J$ Oral Maxillofac Surg 39(4):320-326. https://doi.org/10.1016/j. ijom.2009.11.010

4. Gleeson CF, Patel V, Kwok J, Sproat C (2012) Coronectomy practice. Paper 1. Technique and trouble-shooting. Br J Oral Maxillofac Surg 50(8):739-744. https://doi.org/10.1016/j.bjoms.2012.01.001

5. O'Riordan BC (2004) Coronectomy (intentional partial odontectomy of lower third molars). Oral Surg Oral Med Oral Pathol Oral Radiol Endod 98(3):274-280. https://doi.org/10. 1016/S1079210404000496

6. Sencimen M, Ortakoglu K, Aydin C, Aydintug YS, Ozyigit A, Ozen T, Gunaydin Y (2010) Is endodontic treatment necessary during coronectomy procedure. J Oral Maxillofac Surg 68(10): 2385-2390. https://doi.org/10.1016/j.joms.2010.02.024

7. Knutsson K, Lysell L, Rohlin M (1989) Postoperative status after partial removal of the mandibular third molar. Swed Dent J 13(1-2): $15-22$

8. Gülicher D, Gerlach KL (2001) Sensory impairment of the lingual and inferior alveolar nerves following removal of impacted mandibular third molars. Int J Oral Maxillofac Surg 30(4):306-312

9. Pogrel MA, Lee JS, Muff DF (2004) Coronectomy: a technique to protect the inferior alveolar nerve. J Oral Maxillofac Surg 62(12): $1447-1452$

10. Ecuyer J, Debien J (1984) Surgical deductions. Actualités OdontoStomatologiques 38(148):695-702

11. Renton T, Hankins M, Sproate C, McGurk M (2005) A randomised controlled clinical trial to compare the incidence of injury to the inferior alveolar nerve as a result of coronectomy and removal of mandibular third molars. Br J Oral Maxillofac Surg 43(1):7-12. https://doi.org/10.1016/j.bjoms.2004.09.002

12. Leung YY, Cheung LK (2016) Long-term morbidities of coronectomy on lower third molar. Oral Surg Oral Med Oral Pathol Oral Radiol 121(1):5-11. https://doi.org/10.1016/j.oooo. 2015.07.012

13. Long H, Zhou Y, Liao L, Pyakurel U, Wang Y, Lai W (2012) Coronectomy vs. total removal for third molar extraction: a systematic review. J Dent Res 91(7):659-665. https://doi.org/10.1177/ 0022034512449346

14. Ali AS, Benton JA, Yates JM (2018) Risk of inferior alveolar nerve injury with coronectomy vs surgical extraction of mandibular third molars - a comparison of two techniques and review of the literature. J Oral Rehabil 45(3):250-257. https://doi.org/10.1111/joor. 12589

15. Devine M, Hirani M, Durham J, Nixdorf DR, Renton T (2018) Identifying criteria for diagnosis of post-traumatic pain and altered sensation of the maxillary and mandibular branches of the trigeminal nerve: a systematic review. Oral Surg Oral Med Oral Pathol Oral Radiol 125(6):526-540. https://doi.org/10.1016/j.oooo.2017. 12.020

16. Rolke R, Magerl W, Campbell KA, Schalber C, Caspari S, Birklein F, Treede RD (2006) Quantitative sensory testing: a comprehensive protocol for clinical trials. Eur J Pain 10(1):77-88. https://doi.org/ 10.1016/j.ejpain.2005.02.003

17. Wang Y, Mo X, Zhang J, Fan Y, Wang K, Peter S (2018) Quantitative sensory testing (QST) in the orofacial region of healthy Chinese: influence of site, gender and age. Acta Odontol Scand 76(1):58-63. https://doi.org/10.1080/00016357.2017. 1383511

18. Zhou P, Chen Y, Zhang J, Wang K, Svensson P (2018) Quantitative sensory testing for assessment of somatosensory function in human oral mucosa: a review. Acta Odontol Scand 76(1):13-20. https:// doi.org/10.1080/00016357.2017.1375554

19. Yekta SS, Koch F, Grosjean MB, Esteves-Oliveira M, Stein JM, Ghassemi A, Riediger D, Lampert F, Smeets R (2010) Analysis of 
trigeminal nerve disorders after oral and maxillofacial intervention. Head Face Med 6:24. https://doi.org/10.1186/1746-160X-6-24

20. Said-Yekta S, Smeets R, Esteves-Oliveira M, Stein JM, Riediger D, Lampert F (2012) Verification of nerve integrity after surgical intervention using quantitative sensory testing. J Oral Maxillofac Surg 70(2):263-271. https://doi.org/10.1016/j.joms.2011.03.065

21. Baad-Hansen L, Pigg M, Ivanovic SE, Faris H, List T, Drangsholt M, Svensson P (2013) Intraoral somatosensory abnormalities in patients with atypical odontalgia - a controlled multicenter quantitative sensory testing study. Pain 154(8):1287-1294. https://doi. org/10.1016/j.pain.2013.04.005

22. Hartmann A, Welte-Jzyk C, Seiler M, Daublander M (2017) Neurophysiological changes associated with implant placement. Clin Oral Implants Res 28(5):576-581. https://doi.org/10.1111/clr. 12837

23. Hartmann A, Welte-Jzyk C, Seiler M, Daublander M (2017) Neurophysiological changes associated with implant-associated augmentation procedures in the lower jaw. Clin Implant Dent Relat Res 19(4):725-732. https://doi.org/10.1111/cid.12500

24. Juhl GI, Svensson P, Norholt SE, Jensen TS (2006) Long-lasting mechanical sensitization following third molar surgery. J Orofac Pain 20(1):59-73

25. Juhl GI, Jensen TS, Norholt SE, Svensson P (2008) Central sensitization phenomena after third molar surgery: a quantitative sensory testing study. Eur J Pain 12(1):116-127. https://doi.org/10.1016/j. ejpain.2007.04.002

26. Patel V, Gleeson CF, Kwok J, Sproat C (2013) Coronectomy practice. Paper 2: complications and long term management. Br J Oral Maxillofac Surg 51(4):347-352. https://doi.org/10.1016/j.bjoms. 2012.06.008

27. Rolke R, Baron R, Maier C, Tölle TR, Treede RD, Beyer A, Binder A, Birbaumer N, Birklein F, Bötefür IC, Braune S, Flor H, Huge V, Klug R, Landwehrmeyer GB, Magerl W, Maihöfner C, Rolko C, Schaub C, Scherens A, Sprenger T, Valet M, Wasserka B (2006) Quantitative sensory testing in the German Research Network on Neuropathic Pain (DFNS): standardized protocol and reference values. Pain 123(3):231-243. https://doi.org/10.1016/j.pain.2006. 01.041

28. Yang G, Baad-Hansen L, Wang K, Xie QF, Svensson P (2014) A study on variability of quantitative sensory testing in healthy participants and painful temporomandibular disorder patients. Somatosens Mot Res 31(2):62-71. https://doi.org/10.3109/ 08990220.2013 .869493

29. Yang G, Baad-Hansen L, Wang K, Fu K, Xie QF, Svensson P (2016) Somatosensory abnormalities in Chinese patients with painful temporomandibular disorders. J Headache Pain 17:31. https:// doi.org/10.1186/s10194-016-0632-y

30. Pigg M, Baad-Hansen L, Svensson P, Drangsholt M, List T (2010) Reliability of intraoral quantitative sensory testing (QST). Pain 148(2):220-226. https://doi.org/10.1016/j.pain.2009.10.024

31. Matos R, Wang K, Jensen JD, Jensen T, Neuman B, Svensson P, Arendt-Nielsen L (2011) Quantitative sensory testing in the trigeminal region: site and gender differences. J Orofac Pain 25(2):161169

32. Svensson P, Baad-Hansen L, Pigg M, List T, Eliav E, Ettlin D, Michelotti A, Tsukiyama Y, Matsuka Y, Jääskeläinen SK, Essick G, Greenspan JD, Drangsholt M, Special Interest Group of OroFacial Pain (2011) Guidelines and recommendations for assessment of somatosensory function in oro-facial pain conditions-a taskforce report. J Oral Rehabil 38(5):366-394. https://doi.org/10. 1111/j.1365-2842.2010.02196.x

33. Yang G, Luo Y, Baad-Hansen L, Wang K, Arendt-Nielsen L, Xie QF, Svensson P (2013) Ethnic differences in oro-facial somatosensory profiles-quantitative sensory testing in Chinese and Danes. J Oral Rehabil 40(11):844-853. https://doi.org/10.1111/joor.12091
34. Yang G, Baad-Hansen L, Wang K, Xie QF, Svensson P (2014) Effect of negative emotions evoked by light, noise and taste on trigeminal thermal sensitivity. J Headache Pain 15:71. https://doi. org/10.1186/1129-2377-15-71

35. Kothari SF, Baad-Hansen L, Oono Y, Svensson P (2015) Somatosensory assessment and conditioned pain modulation in temporomandibular disorders pain patients. Pain 156(12):25452555. https://doi.org/10.1097/j.pain.0000000000000325

36. Maier C, Baron R, Tölle TR, Binder A, Birbaumer N, Birklein F, Gierthmühlen J, Flor H, Geber C, Huge V, Krumova EK, Landwehrmeyer GB, Magerl W, Maihöfner C, Richter H, Rolke R, Scherens A, Schwarz A, Sommer C, Tronnier V, Uçeyler N, Valet M, Wasner G, Treede RD (2010) Quantitative sensory testing in the German Research Network on Neuropathic Pain (DFNS): somatosensory abnormalities in 1236 patients with different neuropathic pain syndromes. Pain 150(3):439-450. https://doi.org/10. 1016/j.pain.2010.05.002

37. Larrazábal C, García B, Peñarrocha M, Peñarrocha M (2010) Influence of oral hygiene and smoking on pain and swelling after surgical extraction of impacted mandibular third molars. J Oral Maxillofac Surg 68(1):43-46. https://doi.org/10.1016/j.joms.2009. 07.061

38. Dezawa K, Noma N, Watanabe K, Sato Y, Kohashi R, Tonogi M, Heir G, Eliav E, Imamura Y (2016) Short-term effects of orthognathic surgery on somatosensory function and recovery pattern in the early postoperative period. J Oral Sci 58(2):177-184. https://doi.org/10.2334/josnusd.15-0670

39. Huang C, Zou W, Lee K, Wang E, Zhu X, Guo Q (2012) Different symptoms of neuropathic pain can be induced by different degrees of compressive force on the C7 dorsal root of rats. Spine J 12(12): 1154-1160. https://doi.org/10.1016/j.spinee.2012.10.036

40. Luo Y, Svensson P, Jensen JD, Jensen T, Neuman B, ArendtNielsen L, Wang K (2014) Quantitative sensory testing in patients with or without ongoing pain one year after orthognathic surgery. J Oral Facial Pain Headache 28(4):306-316

41. Kim HK, Kim KS, Kim ME (2017) Thermal perception as a key factor for assessing effects of trigeminal nerve injury. J Oral Facial Pain Headache 31(2):129-138

42. Kramer S, Baeumler P, Geber C, Fleckenstein J, Simang M, Haas L, Schober G, Pfab F, Treede RD, Irnich D (2018) Somatosensory profiles in acute herpes zoster and predictors of postherpetic neuralgia. Pain. https://doi.org/10.1097/j.pain.0000000000001467

43. Hillerup S, Stoltze K (2007) Lingual nerve injury II. Observations on sensory recovery after micro-neurosurgical reconstruction. Int $\mathrm{J}$ Oral Maxillofac Surg 36(12):1139-1145. https://doi.org/10.1016/j. ijom.2007.06.005

44. Martinez V, Üçeyler N, Ben Ammar S, Alvarez JC, Gaudot F, Sommer C, Bouhassira D, Fletcher D (2015) Clinical, histological, and biochemical predictors of postsurgical neuropathic pain. Pain 156(11):2390-2398. https://doi.org/10.1097/j.pain. 0000000000000286

45. Teerijoki-Oksa T, Jaaskelainen S, Forssell K, Virtanen A, Forssell H (2003) An evaluation of clinical and electrophysiologic tests in nerve injury diagnosis after mandibular sagittal split osteotomy. Int J Oral Maxillofac Surg 32(1):15-23. https://doi.org/10.1054/ ijom.2002.0325

46. Wagner ME, Rana M, Traenkenschuh W, Kokemueller H, Eckardt AM, Gellrich NC (2011) Piezoelectric-assisted removal of a benign fibrous histiocytoma of the mandible: an innovative technique for prevention of dentoalveolar nerve injury. Head Face Med 7(20). https://doi.org/10.1186/1746-160X-7-20

47. Leung YY, Cheung LK (2012) Coronectomy of the lower third molar is safe within the first 3 years. J Oral Maxillofac Surg 70(7):1515-1522. https://doi.org/10.1016/j.joms.2011.12.029

48. Vignudelli E, Monaco G, Mazzoni A, Marchetti C (2015) Root fragment vitality after coronectomy: histological evidence in a case. 
J Oral Maxillofac Surg 73(11):2093.e1-2093.e5. https://doi.org/10. 1016/j.joms.2015.06.179

49. Dalle Carbonare M, Zavattini A, Duncan M, Williams M, Moody A (2017) Injury to the inferior alveolar and lingual nerves in successful and failed coronectomies: systematic review. Br J Oral Maxillofac Surg 55(9):892-898. https://doi.org/10.1016/j.bjoms. 2017.09.006

50. Porporatti AL, Bonjardim LR, Stuginski-Barbosa J, Bonfante EA, Costa YM, Rodrigues Conti PC (2017) Pain from dental implant placement, inflammatory pulpitis pain, and neuropathic pain present different somatosensory profiles. J Oral Facial Pain Headache 31(1):19-29

51. Treede RD, Meyer RA, Raja SN, Campbell JN (1992) Peripheral and central mechanisms of cutaneous hyperalgesia. Prog Neurobiol 38(4):397-421
52. Cardoso CL, Rodrigues MT, Ferreira Júnior O, Garlet GP, de Carvalho PS (2010) Clinical concepts of dry socket. J Oral Maxillofac Surg 68(8):1922-1932. https://doi.org/10.1016/j.joms. 2009.09.085

53. Leung YY, Cheung KY (2018) Root migration pattern after third molar coronectomy: a long-term analysis. Int J Oral Maxillofac Surg 47(6):802-808. https://doi.org/10.1016/j.ijom.2018.01.015

54. Yeung A, Wong N, Bornstein MM, Leung YY (2018) Threedimensional radiographic evaluation of root migration patterns 48.5 years after lower third molar coronectomy: a cone beam computed tomography study. Int J Oral Maxillofac Surg. https://doi.org/ 10.1016/j.ijom.2018.03.014

Publisher's note Springer Nature remains neutral with regard to jurisdictional claims in published maps and institutional affiliations. 